\title{
A computer-assisted model for detection of MRI signs of Crohn's disease activity: future or fiction?
}

\author{
Jeroen A.W. Tielbeek, ${ }^{1}$ Frans M. Vos, ${ }^{1,2}$ Jaap Stoker ${ }^{1}$ \\ ${ }^{1}$ Department of Radiology, Academic Medical Center, University of Amsterdam, Meibergdreef 9, 1105 AZ Amsterdam, \\ The Netherlands \\ ${ }^{2}$ Quantitative Imaging Group, Department of Imaging Science and Technology, Delft University of Technology, \\ Lorentzweg 1, 2628 CJ Delft, The Netherlands
}

\begin{abstract}
Magnetic resonance imaging is increasingly used for abdominal evaluation and is more and more considered as the optimal imaging technique for detection of mural inflammation in patients with Crohn's disease. Grading the disease activity is important in daily clinical practice to monitor the medical treatment and is assessed by evaluating different magnetic resonance imaging features. Unfortunately, only moderate interobserver agreement is reported for most of the subjective features and should be improved. A computer-assisted model for automatic detection of abnormalities, ability to grade disease severity, and thereby influence clinical disease management based on magnetic resonance imaging is missing. Recent techniques have focused on semi-automated methods for classification and segmentation of the bowel and also on objective measurement of bowel wall enhancement using absolute T1-values or dynamic contrast-enhanced imaging. This article reviews the available computerized techniques, as well as preferred developments.
\end{abstract}

Key words: Crohn's disease-Magnetic resonance imaging - Intestinal imaging-Disease activity-Computer assisted model

Abdominal magnetic resonance imaging (MRI) is widely used for diagnosing and grading luminal Crohn's disease (CD). It typically involves a luminal (oral) and an

Correspondence to: Jeroen A.W. Tielbeek; email: j.a.w.tielbeek@, amc.uva.nl intravenous contrast medium in order to combine transmural and extra-intestinal evaluation of disease activity. Grading disease activity is important in daily clinical practice to monitor the often costly and burdensome medical treatment and define treatment strategies. In addition, pharmaceutical trials increasingly employ MRI as outcome measure.

Conventionally, disease activity is assessed by evaluating the presence of mural changes (e.g., wall enhancement, bowel wall thickening, wall edema, and stratification), stenoses, length of the involved segments, skip lesions and complications (e.g., fistulas) [1-7]. In fact, almost all articles on MRI in luminal CD use these MRI features for assessment of disease activity $[8,9]$ and these features are considered most important by international experts [10].

However, grading the disease activity based on these MRI features has intrinsic limitations related to restrictions of the MRI technique. Also, it is a subjective evaluation while varying weight is attributed to these features. For instance, Jensen et al. [11] found only moderate interobserver agreement $(\mathrm{kappa}=0.48)$ in detection of CD with MR enterography. Another recent study by Ziech et al. [12] reported a weak to moderate interobserver agreement for most of the subjective MRI features. This is in accordance with other studies comparing interobserver variability of subjective MRI features $[13,14]$. A questionnaire among international research groups showed that different weight is given to certain features and different grading systems are used, hampering comparison of study results [10]. For example, some reviews $[15,16]$ presented absolute contrast enhancement as a marker of disease activity, while others dispute this [7, 17]. Based on the present methods of grading, MRI has been shown to be accurate for severe 
disease (in $91 \%$ of cases accurate grading), but mediocre for mild disease or remission (accurate grading in only $62 \%$ of cases) [18].

In a recent study Rimola et al. [19] used multivariate analyses correlating radiological features to the Crohn's disease endoscopic index of severity (CDEIS). The independent variables included in this regression analysis were those with statistical significance (i.e., $P<0.05$ ) in a univariate test. As such, the regression analysis rendered objectively determined weights that were attributed to the independent variables in a Magnetic resonance index of activity (MaRIA). This MaRIA score had a high-diagnostic accuracy both in a derivation cohort and an independent validation cohort for diagnosis of active CD [20]. The study confirmed the radiologic changes that should be evaluated to that end, which were wall thickening, wall signal intensity, relative contrast enhancement, the presence of edema, ulcers, enlarged lymph nodes, and pseudopolyps.

Clearly, a system is preferred that renders a fine grading of the disease severity for accurate treatment monitoring. Further, for surgical treatment the location of the disease and the length of the involved segments are important as well. At present the radiologist's reading of the length of involved segments is relatively cumbersome and no data are available on the accuracy and the precision.

For an optimal evaluation of response monitoring, MRI should be a robust, objective and reproducible technique. Applying a (semi-)automated method might improve the interobserver variation and allow a finer diagnosis scale as compared to the gross scale (remission-mild-severe) used by the radiologist. Therefore, development of computer-assisted diagnosis tools for quantitative image-based analysis of $\mathrm{CD}$ is pivotal. This article aims at reviewing the semi-automated techniques that are currently available for assessment of CD severity.

\section{Semi-automated methods in Crohn's disease}

The literature on semi-automated methods in CD is very sparse. Much work that has been done on (semi-)automated methods related to imaging of the gastrointestinal tract concerns CT colonography. Specifically, over the past years many (semi-)automated techniques for CT colonography were developed such as sophisticated techniques for visualization of the bowel surface [21], electronic bowel cleansing [22], and computer aided detection [23] in CT colonography. These techniques proved helpful and have led to the use of these techniques in daily practice.

One might expect that such methods can be used in MRI of Crohn's disease as well. However, in contrast to the values in CT images, which represent well reproducible CT numbers, values in MR images depend on a large number of variables and have no absolute meaning. Specifically, MRI images are influenced by signal fluctuations that emanate both from global (bias field) as well as local effects (heterogeneities in tissue). The resulting variation in signal value precludes simple approaches (e.g., thresholding) for MRI data. Presumably, the complexity of processing MR data has resulted in a limited number of practically used and studied techniques.

One study reports on automated methods that take into account multiple imaging features to identify small bowel loops (segmentation) and to quantify aspects of disease severity (feature measurement) [8, 24]. A 2D steady-state free precession (FIESTA) scan was used in combination with a fat suppressed scan to correctly segment the bowel. Registration of the scans was needed, because of patient motion and temporal movement of the bowel. The FIESTA scans served as input for a Gaussian classifier which tentatively divided the dataset into different components: fat, muscle/background and enhancing bowel. The remaining bowel regions were finally segmented on the fat suppressed scan. The operator could then select the objects of interest and label them as enhancing bowel. In the end, this might help to objectively assess inflammation, and determine its multifocality, severity, length, and radial involvement.

Unfortunately, complete automation seemed to be not feasible due to the variability in the intensity values and significant artefacts that emanated from the imaging process. The proposed image processing methods were designed to facilitate the evaluation process and reduce the effort required by the radiologist to a few minutes. The registration, classification, and segmentation might be further automated by the application of prior knowledge and heuristic rules [24]. Therefore, additional research is required.

Another development concerns objective measurements of enhancement. Pathological increase in bowel wall T1-signal intensity (enhancement) after intravenous (IV) administration of Gadolinium is an important characteristic of active CD (see above) [2, 10]. The increase of the longitudinal relaxation rate $(1 / \mathrm{T} 1)$ is directly proportional to the tissue concentration of the contrast medium [26]. Therefore, calculation of the absolute T1-values in tissue before and after IV injection of Gadolinium would provide objective measurements of the Gadolinium-uptake. Inter- and intraobserver variability of manual measurement of the bowel wall enhancement is reported to be poor, but improving after choosing fixed regions-of-interest (ROI) [15, 27]. As such, bowel wall enhancement may be assessed in a more objective manner.

Alternatively, methods have been evaluated to objectively score disease activity in patients with $\mathrm{CD}$ using features of the uptake curves from dynamic contrast-enhanced (DCE-) MRI [1]. The slope of 

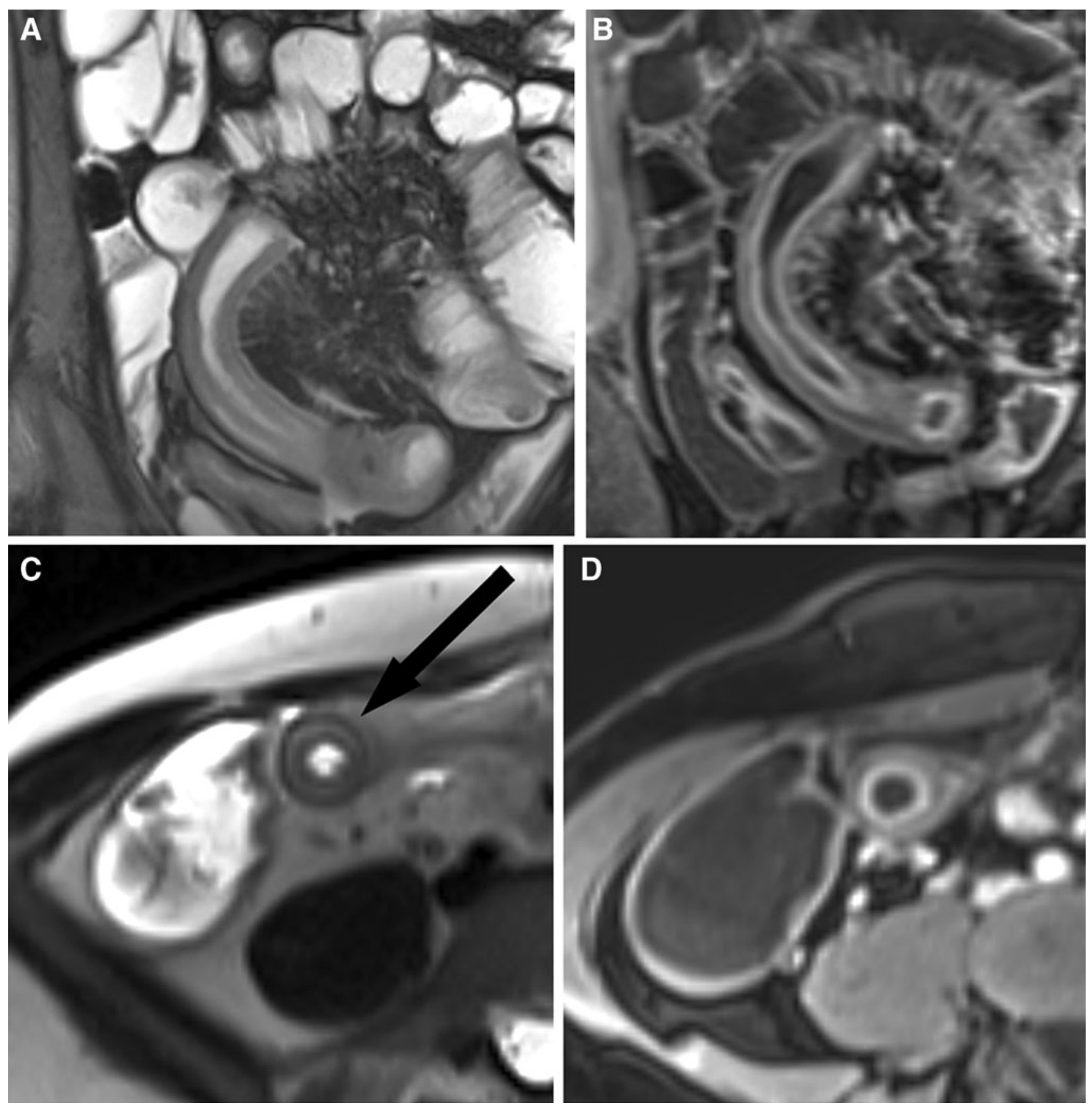

Fig. 1. Forty-two-year-old female patient with Crohn's disease who previously underwent an ileocecal resection. A Coronal balanced steady-state free precession (true-FISP) image with fat saturation, $\mathbf{B}$ coronal T1-weighted volume interpolated breath hold imaging (VIBE) post contrast image with fat saturation, C transverse Half-Fourier single shot RARE (HASTE) image, and D transverse T1-weighted VIBE post contrast image with fat saturation shows bowel wall thickening and post contrast mucosal wall enhancement. On

enhancement, a semi-quantitative parameter, has correlated negatively with microvessel density and positively with disease chronicity suggesting that chronic low perfusion may stimulate new vessel formation [28]. Semi- the HASTE sequence high signal intensity of the bowel wall due to oedema is visible (arrow), it could also be related to fat in the wall but based on the other sequences this could be excluded (use of (additional) fat saturation HASTE sequence is preferable; see Fig. 2E). The mucosal wall enhancement and the high signal intensity on T2 (HASTE) are features indicating acute disease activity. The length of the affected segment is approximately $40 \mathrm{~cm}$.

quantitative parameters such as curve shape have also been found to correlate with disease score in perianal CD [29]. All such quantitative parameters are in themselves reviewer-independent. 


\section{Discussion}

There is a clear demand for semi-automated evaluation of MR images in CD. Currently, a computer-assisted diagnosis tool for automatic detection of abnormalities, ability to grade disease severity, and thereby influence clinical disease management based on MRI is missing. Development of such a system is a complex task, particularly due to the signal fluctuation inherent to MRI. Moreover, the limited thickness of the bowel wall and the presence of peristalsis further complicates the development of new techniques. A combination of (semi-)automated segmentation and different registration techniques to identify, respectively, align regions of interest in MRI images would be extremely useful. This should facilitate the measurement of descriptive properties of CD activity in the images and the application of pattern recognition techniques to detect and rate abnormalities. In turn, the latter would support the establishment of a combined, objective, and quantitative disease severity index.

It may be anticipated that such tools requires several technical developments.

An important step in the development of a computerassisted model might be the (semi-)automated identification of the bowel surface. Initially, a radiologist could manually select a region in an intestinal segment where CD activity is expected. Subsequently, the model would locally identify the bowel wall, from which the wall thickness and enhancement could be deduced. In addition, the model might give the length of the affected part of the segment. The model would assist the radiologist in this way by objectively quantifying features of the affected bowel segment, which in itself already could be very useful in the follow-up of patients.

Fully automated object detection (detection of diseased regions without user interaction) is considered a very difficult task in the context of MR imaging, which may not be achieved shortly. Clearly, such a system would be favourable in the future, though.

Registration of the different MRI images to have spatially corresponding data might be a crucial step in the development of a computer-assisted model [24]. This would facilitate a combined assessment of multiple MRI features (bowel wall thickness, signal intensity on T2weighted images, enhancement after intravenous contrast and stratification) from different sequences. As such, it may provide the radiologist a better overview of the location and characteristics of the abnormalities in the GI tract. For example, to determine whether an intestinal segment is fibrotic or not, a radiologist has to evaluate the signal intensity and stratification of the bowel wall on a post contrast T1-weighted sequence and a T2-weighted sequence (Figs. 1, 2).

Based on the aforementioned features (e.g., bowel wall thickness, signal intensity on fat sat $\mathrm{T} 2$, layered enhancement), a sophisticated pattern recognition
Fig. 2. The same Crohn's disease patient as in Fig. 1; nine months later after treatment with adalimumab (HUMIRA, Abbott). A Coronal true-FISP image, B coronal T1-weighted VIBE post contrast image, $\mathbf{C}$ transverse HASTE image, D transverse T1-weighted VIBE post contrast image, $\mathbf{E}$ axial HASTE image with fat saturation and $\mathbf{F}$ an axial true-FISP sequence shows wall thickening and still increased enhancement. Edema has decreased as compared to Fig. 1, indicating less active disease. Prominent engorged vasa recta in the mesentery (comb sign) are also identified (asterisk). The length of the affected segment remains approximately $40 \mathrm{~cm}$. Mapping different sequences by ICT tools would give the radiologist a better overview of the same segment, and a better insight in differentiating between fibrosis and inflammation. Further, objective measurement of the length and/or wall enhancement would help the radiologist and gastroenterologist in therapy monitoring.

technique might automatically compute the probability that the segment is affected by $\mathrm{CD}$, and even determine if it is fibrosed and/or inflamed. Subsequently, the radiologist could be informed about the characteristics of the segment at a glance.

Eventually, the tool could even indicate the grade of inflammation of the segment by means of visualization techniques thereby improving the perception and accelerating the recognition time [30].

A further highly needed technical development will be to combine MR, laboratory (e.g., C-reactive protein) and clinical data (e.g., the Harvey Bradshaw index [31] or Crohn's Disease activity index [32]) for a more reliable and precise diagnostic performance. The combination of quantitative imaging and clinical biomarkers of disease will activity draw on the strengths of both methodologies and it is anticipated that it will add to the robustness of any predictive model.

Moreover, a visualization software toolbox could enable interactive visualization of the bowel and the bowel wall characteristics. It will feature techniques for concurrent visualization of the multiscale clinical patient data as well as the properties measured by image analysis and classification.

Finally, it is expected that patient education will be supported by developed visualization techniques. Patient education is becoming an increasingly important aspect of medical care. Illustrations can be a valuable aid in explaining diagnoses and treatments [33]. Recent progress [34] in the area of illustrative visualization seeks to reproduce the expressiveness and comprehensibility of traditional illustrations using advanced visualization techniques.

Perceptually effective visualization techniques as well as methods to evaluate their effectiveness will support this development [30]. Such an approach makes it feasible to generate patient-specific depictions of particular pathologies which are easy to understand for laymen. Similar techniques may also be used to aid medical education and communication [35]. 

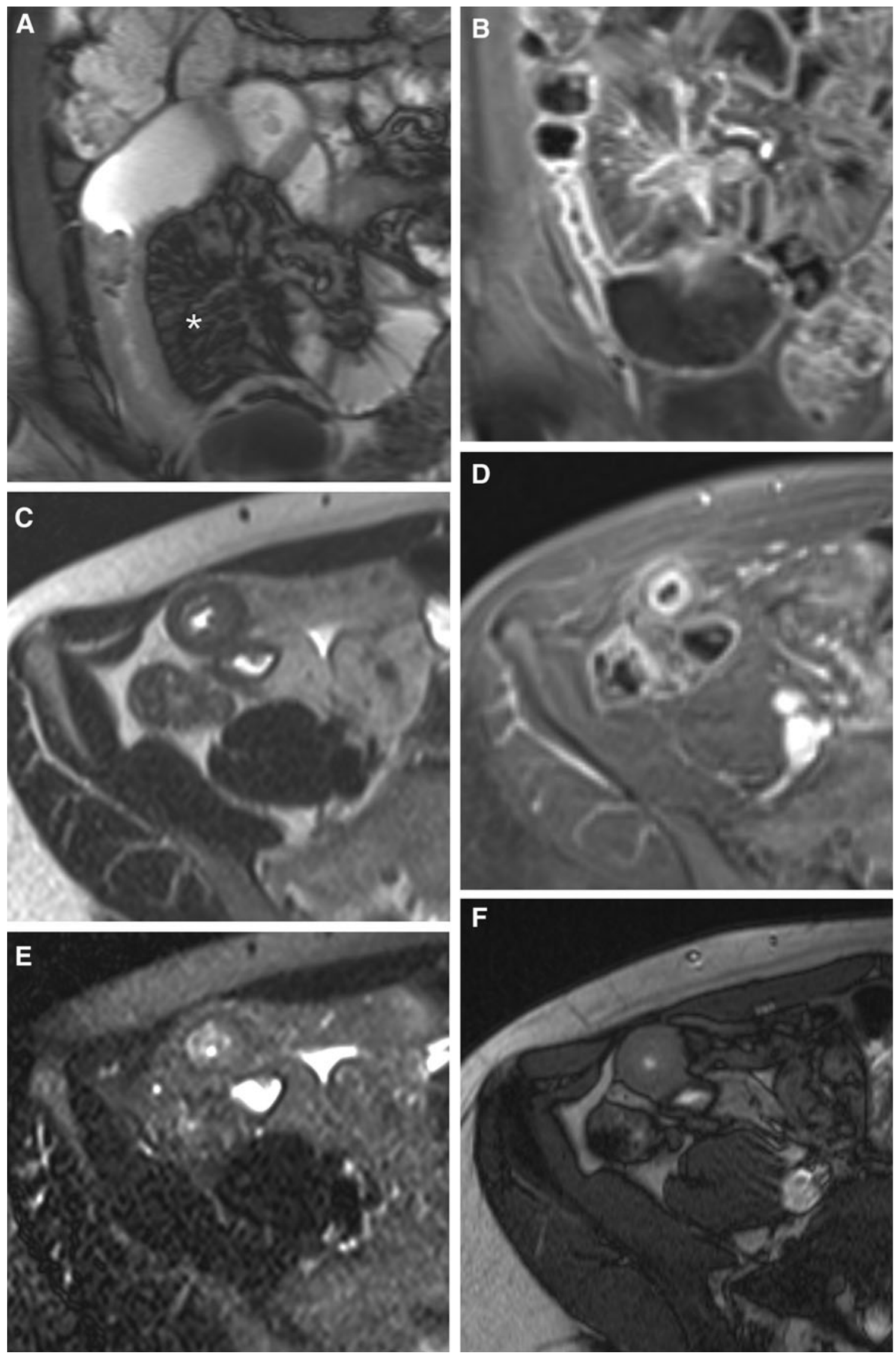


\section{Conclusion}

(Semi-)automated assessment of Crohn's disease is not fiction anymore as already limited tools are available. However, substantial steps have to be made to come to robust (semi-)automated assessment tools. It would be a ground-breaking development if the radiologist could be assisted by automatic detection and quantification of abnormalities at MRI of the gastrointestinal tract and have an objective assessment of disease severity.

To that end ICT tools should be created that extract regions of interest (semi-)automatically from MRI data, analyze the small bowel by use of mural features, use a classifier to predict the presence of active Crohn's disease and the ability to grade disease severity in the near future.

Acknowledgement. The research leading to these results has received funding from the European Community's Seventh Framework Programme (FP7/2007-2013) of the VIGOR $^{++}$Project under Grant Agreement Nr. 270379.

Open Access. This article is distributed under the terms of the Creative Commons Attribution Noncommercial License which permits any noncommercial use, distribution, and reproduction in any medium, provided the original author(s) and source are credited.

\section{References}

1. Florie J, Wasser MNJM, Arts-Cieslik K, et al. (2006) Dynamic contrast-enhanced MRI of the bowel wall for assessment of disease activity in Crohn's disease. AJR 186:1384-1392

2. Koh DM, Miao Y, Chinn RJS, et al. (2001) MR imaging evaluation of the activity of Crohn's disease. AJR 177:1325-1332

3. Shoenut JP, Semelka RC, Silverman R, Yaffe CS, Micflikier AB (1993) Magnetic resonance imaging in inflammatory bowel disease. J Clin Gastroenterol 17:73-78

4. Shoenut JP, Semelka RC, Magro CM, et al. (1994) Comparison of magnetic resonance imaging and endoscopy in distinguishing the type and severity of inflammatory bowel disease. J Clin Gastroenterol 19:31-35

5. Maccioni F, Viscido A, Broglia L, et al. (2000) Evaluation of Crohn disease activity with magnetic resonance imaging. Abdom Imaging 25:219-228

6. Maccioni F, Viscido A, Marini M, Caprilli R (2002) MRI evaluation of Crohn's disease of the small and large bowel with the use of negative superparamagnetic oral contrast agents. Abdom Imaging 27:384-393

7. Punwani S, Rodriguez-Justo M, Bainbridge A, et al. (2009) Mural inflammation in Crohn disease: location-matched histologic validation of MR imaging features. Radiology 252:712-720

8. Fletcher JG, Fidler JL, Bruining DH, Huprich JE (2011) New concepts in intestinal imaging for inflammatory bowel diseases. Gastroenterology 140:1795-1806

9. Horsthuis K, Bipat S, Bennink RJ, Stoker J (2008) Inflammatory bowel disease diagnosed with US, MR, scintigraphy, and CT: metaanalysis of prospective studies. Radiology 247:64-79

10. Ziech MLW, Bossuyt PMM, Laghi A et al. (2011) Grading luminal Crohn's disease: which MRI features are considered as important? Eur J Radiol. doi:10.1016/j.ejrad.2011.05.033

11. Jensen MD, Ormstrup T, Vagn-Hansen C, Østergaard L, Rafaelsen SR (2011) Interobserver and intermodality agreement for detection of small bowel Crohn's disease with MR enterography and CT enterography. Inflamm Bowel Dis 17:1081-1088

12. Ziech MLW, Bipat S, Roelofs JJTH, et al. (2011) Retrospective comparison of magnetic resonance imaging features and histopathology in Crohn's disease patients. Eur J Radiol. doi:10.1016/ j.ejrad.2010.12.075
13. Florie J, Horsthuis K, Hommes DW, et al. (2005) Magnetic resonance imaging compared with ileocolonoscopy in evaluating disease severity in Crohn's disease. Clin Gastroenterol Hepatol 3: $1221-1228$

14. van Gemert-Horsthuis K, Florie J, Hommes DW, et al. (2006) Feasibility of evaluating Crohn's disease activity at 3.0 Tesla. J Magn Reson Imaging 24:340-348

15. Sharman A, Zealley IA, Greenhalgh R, Bassett P, Taylor SA (2009) MRI of small bowel Crohn's disease: determining the reproducibility of bowel wall gadolinium enhancement measurements. Eur Radiol 19:1960-1967

16. Gourtsoyiannis NC, Papanikolaou N, Karantanas A (2006) Magnetic resonance imaging evaluation of small intestinal Crohn's disease. Best Pract Res Clin Gastroenterol 20:137-156

17. Tolan DJM, Greenhalgh R, Zealley IA, Halligan S, Taylor SA (2010) MR enterographic manifestations of small bowel Crohn disease. Radiographics 30:367-384

18. Horsthuis K, Bipat S, Stokkers PCF, Stoker J (2009) Magnetic resonance imaging for evaluation of disease activity in Crohn's disease: a systematic review. Eur Radiol 19:1450-1460

19. Rimola J, Rodriguez S, García-Bosch O, et al. (2009) Magnetic resonance for assessment of disease activity and severity in ileocolonic Crohn's disease. Gut 58:1113-1120

20. Rimola J, Ordás I, Rodriguez S, et al. (2011) Magnetic resonance imaging for evaluation of Crohn's disease: validation of parameters of severity and quantitative index of activity. Inflamm Bowel Dis 17:1759-1768

21. Vos FM, van Gelder RE, Serlie IWO, et al. (2003) Three-dimensional display modes for CT colonography: conventional 3D virtual colonoscopy versus unfolded cube projection. Radiology 228:878885

22. Serlie IWO, de Vries AH, van Vliet LJ, et al. (2008) Lesion conspicuity and efficiency of CT colonography with electronic cleansing based on a three-material transition model. AJR 191:14931502

23. Bielen D, Kiss G (2007) Computer-aided detection for CT colonography: update. Abdom imaging 32:571-581

24. Holmes D 3rd, Huprich J, Fidler J, Robb R, Fletcher J (2010) Feasibility of developing interactive small bowel segmentation from MR enterography. MICCAI workshop: Virtual Colonoscopy \& Abdominal Imaging 105-110. http://www.virtualcolonmiccai2010.org/Proceedings/MICCAI2010-VCWorkshop-Proceed ings.pdf

25. Pupillo VA, Di Cesare E, Frieri G, et al. (2007) Assessment of inflammatory activity in Crohn's disease by means of dynamic contrast-enhanced MRI. Radiol Med 112:798-809

26. Gowland P, Mansfield P, Bullock P, et al. (1992) Dynamic studies of gadolinium uptake in brain tumors using inversion-recovery echo-planar imaging. Magn Reson Med 26:241-258

27. Horsthuis K, Nederveen AJ, de Feiter M-W, et al. (2009) Mapping of T1-values and Gadolinium-concentrations in MRI as indicator of disease activity in luminal Crohn's disease: a feasibility study. J Magn Reson Imaging 29:488-493

28. Taylor SA, Rodriguez-justo M, Bainbridge A, et al. (2009) Mural Crohn disease: correlation of dynamic contrast-enhanced MR imaging findings with angiogenesis and inflammation at histologic examination - pilot study. Radiology 251(2):369-379

29. Horsthuis K, Lavini C, Bipat S, Stokkers PCF, Stoker J (2009) Perianal Crohn disease: evaluation of dynamic contrast-enhanced MR imaging as an indicator of disease activity. Radiology 251:380387

30. Kuß a , Gensel M, Meyer B, Dercksen VJ, Prohaska S (2010) Effective techniques to visualize filament-surface relationships. Comput Graph Forum 29:1003-1012

31. Harvey RF, Bradshaw JM (1980) A simple index of Crohn's-disease activity. Lancet 1:514

32. Best WR, Becktel JM, Singleton JW, Kern F (1976) Development of a Crohn's disease activity index. National cooperative Crohn's disease study. Gastroenterology 70:439-444

33. Houts PS, Doak CC, Doak LG, Loscalzo MJ (2006) The role of pictures in improving health communication: a review of research on attention, comprehension, recall, and adherence. Patient Educ Couns 61:173-190 
34. Bruckner S, Groller ME (2005) VolumeShop: An Interactive System for Direct Volume Illustration Visualization. VIS 05 IEEE 671-678. http://ieeexplore.ieee.org/lpdocs/epic03/wrapper.htm?ar number $=1532856$
35. Ritter F, Hansen C, Dicken V, et al. (2006) Real-time illustration of vascular structures. IEEE Trans Vis Comput Graph 12:877884 